\title{
Pneumocystis pneumonia risk among viral acute respiratory distress syndrome related or not to COVID 19
}

\author{
Keyvan Razazi ${ }^{1,2 \dagger}{ }^{\dagger}$, Romain Arrestier ${ }^{1,2,6^{*}+} \oplus$, Anne Fleur Haudebourg ${ }^{1,2}$, Francoise Botterel ${ }^{3,4 \dagger}$ and \\ Armand Mekontso Dessap ${ }^{1,2,5+}$ and the COVID PCP group
}

Lymphopenia, corticosteroids and immunomodulatory therapeutics frequently used in COVID-19 patients with acute respiratory distress syndrome (C-ARDS) may be contributing factors to opportunistic infection such as Pneumocystis jirovecii pneumonia (PCP).

We conducted a retrospective study to compare the prevalence of PCP between patients with C-ARDS and those with non-SARS-CoV-2 viral ARDS (NC-ARDS).

Methods and some data from this cohort have been previously published [1]. There was no systematic protocol to search for PCP but in case of suspicion of PCP (respiratory symptoms with any consistent radiographic features), several analyses were performed on respiratory samples, such as broncho-alveolar lavage (BAL), blind protected sample, or sputum. It included direct examination (using May-Grünwald Giemsa (MGG), or immunofluorescence staining), detection of Pneumocystis jirovecii DNA by real-time polymerase chain reaction (qPCR) [2], and serum (1-3)- $\beta$-D-glucan. During the COVID-19 outbreak, immunofluorescence staining was not performed. PCP was defined as per the revised EORTC/MSGERC definition [3] as follows: proven in case of suspicion with positive direct examination; possible in case of suspicion with positive $\mathrm{qPCR}$ and positive BDG in $\geq 2$ consecutive

\footnotetext{
*Correspondence: romain.arrestier@aphp.fr

${ }^{\dagger}$ Keyvan Razazi, Romain Arrestier, Francoise Botterel, Armand Mekontso

Dessap have equally contributed to this work

${ }^{1}$ Service de Médecine Intensive Réanimation, AP-HP (Assistance

Publique-Hôpitaux de Paris), Hôpitaux Universitaires Henri Mondor, DMU Médecine, 94010 Créteil, France

Full list of author information is available at the end of the article
}

serum samples provided other etiologies have been excluded. SARS-CoV-2 and other viruses were not considered a priori as host factors. Patients with positive qPCR but lacking the other criteria for possible PCP were classified as colonized.

The primary endpoint was the difference in prevalence of PCP between C-ARDS and NC-ARDS patients.

No statistical sample size calculation was performed a priori, and sample size was equal to the number of patients treated during the study period. All patients were included only once.

Between October 1, 2009, and April 29, 2020, ninety patients had C-ARDS (positive RT PCR test for SARS$\mathrm{CoV}-2$ ), while 82 patients had viral NC-ARDS. Our study comprises 120 patients with fungal analyses on respiratory samples obtained from $81 \mathrm{C}$-ARDS and 39 NC-ARDS patients. NC-ARDS patients had more comorbidities were more often immunocompromised, and had lower lymphocyte counts than C-ARDS patients (Table 1). C-ARDS patient received less steroid than NCARDS patients because they were included before randomized trials demonstrating decreased mortality with dexamethasone.

Pneumocystis analyses were performed on a mean of 3.1 respiratory sample per patient (range 1-15). Direct examination was performed in a total of 72 samples, with two positive cases. qPCR was performed in a total of 368 samples (294 blind protected samples, $72 \mathrm{BAL}$, and three sputum). All qPCR were negative in C-ARDS patients, while five (13\%) NC-ARDS patients had at least original author(s) and the source, provide a link to the Creative Commons licence, and indicate if changes were made. The images or other third party material in this article are included in the article's Creative Commons licence, unless indicated otherwise in a credit line to the material. If material is not included in the article's Creative Commons licence and your intended use is not permitted by statutory regulation or exceeds the permitted use, you will need to obtain permission directly from the copyright holder. To view a copy of this licence, visit http://creativecommons.org/licenses/by/4.0/. The Creative Commons Public Domain Dedication waiver (http://creativeco mmons.org/publicdomain/zero/1.0/) applies to the data made available in this article, unless otherwise stated in a credit line to the data. 
Table 1 Characteristics of patients with Pneumocystis jirovecii research according to C-ARDS and NC-ARDS patients

\begin{tabular}{|c|c|c|c|}
\hline & NC-ARDS $(n=39)$ & C-ARDS $(n=81)$ & p-value \\
\hline Age, median [IQR] & $61.8[56.1-69.3]$ & $58[52-69.5]$ & 0.32 \\
\hline Male gender & $28(72 \%)$ & $65(80 \%)$ & 0.30 \\
\hline \multicolumn{4}{|l|}{ Medical history } \\
\hline Mc Cabe & & & $<0.0001$ \\
\hline No underlying condition & $13(33 \%)$ & $70(86 \%)$ & \\
\hline Ultimately fatal & $16(41 \%)$ & $10(12 \%)$ & \\
\hline Rapidly fatal disease & $10(26 \%)$ & $1(1 \%)$ & \\
\hline Charlson comorbidity index & $3[2-4]$ & $1[0-2]$ & $<0.0001$ \\
\hline Diabetes mellitus & $11(28 \%)$ & $38(47 \%)$ & 0.051 \\
\hline Congestive heart failure (NYHA 3-4) & $3(8 \%)$ & $6(7 \%)$ & $>0.99$ \\
\hline Supraventricular arrhythmia & $5(13 \%)$ & $8(10 \%)$ & 0.76 \\
\hline Hypertension & $16(41 \%)$ & $52(64 \%)$ & 0.016 \\
\hline COPD & $2(5 \%)$ & $8(10 \%)$ & 0.50 \\
\hline Chronic renal failure & $8(21 \%)$ & $13(16 \%)$ & 0.55 \\
\hline Dialysis & $3(8 \%)$ & $2(3 \%)$ & 0.33 \\
\hline Stroke & $1(3 \%)$ & $3(4 \%)$ & $>0.99$ \\
\hline Liver cirrhosis (Child C) & $1(3 \%)$ & $0(0 \%)$ & 0.33 \\
\hline Current smoking & $8(21 \%)$ & $21(26 \%)$ & 0.52 \\
\hline \multicolumn{4}{|l|}{ Immunosuppression conditions } \\
\hline Overall & $32(82 \%)$ & $13(16 \%)$ & $<0.0001$ \\
\hline Solid cancer & $2(5 \%)$ & $4(5 \%)$ & $>0.99$ \\
\hline Blood cancer & $15(38 \%)$ & $0(0 \%)$ & $<0.0001$ \\
\hline Organ transplant & $9(23 \%)$ & $5(6 \%)$ & 0.013 \\
\hline HIV infection & $3(8 \%)$ & $3(4 \%)$ & 0.39 \\
\hline Sickle cell disease & $1(3 \%)$ & $3(4 \%)$ & $>0.99$ \\
\hline Others & $4(10 \%)$ & $1(1 \%)$ & 0.038 \\
\hline \multicolumn{4}{|c|}{ Clinical characteristics upon ICU admission } \\
\hline IGS2 & $51[37-68]$ & 35 [27-43] & $<0.0001$ \\
\hline Baseline SOFA, median [IQR] & $9[6-12]$ & $7[4-8]$ & $<0.0001$ \\
\hline ARDS classification (Berlin definition) & & & 0.046 \\
\hline Mild & $12(31 \%)$ & $10(12 \%)$ & \\
\hline Moderate & $18(46 \%)$ & $44(54 \%)$ & \\
\hline Severe & $9(23 \%)$ & $27(33 \%)$ & \\
\hline Norepinephrine, n (\%) & $20(51.3 \%)$ & $35(43.2 \%)$ & 0.41 \\
\hline Serum creatinine $(\mu \mathrm{mol} / \mathrm{L})$ & $147[83-226]$ & $82[66-124]$ & 0.001 \\
\hline White blood cell count $\left(\times 10^{9} / \mathrm{L}\right)$ & $5.4[3-14.8]$ & $8.1[5.5-11.9]$ & 0.44 \\
\hline Lymphocyte count $\left(\times 10^{9} / \mathrm{L}\right)$ & $0.4[0.2-0.9]$ & $0.8[0.5-1.2]$ & 0.01 \\
\hline Documented bacterial co-infections & $18(46 \%)$ & $13(16 \%)$ & $<0.0001$ \\
\hline \multicolumn{4}{|l|}{ Treatments during the first $24 \mathrm{~h}$} \\
\hline Antibiotics & $39(100 \%)$ & $81(100 \%)$ & $>0.99$ \\
\hline Antiviral treatment & $26(67 \%)$ & $65(80 \%)$ & 0.10 \\
\hline Corticosteroids (any dose) & $21 / 38(55 \%)$ & $10 / 79(13 \%)^{*}$ & $<0.0001$ \\
\hline Corticosteroids (low dose) & 20/38 (53\%) & $8 / 79(10 \%)^{*}$ & $<0.0001$ \\
\hline Corticosteroids (high dose) \# & $1 / 38(3 \%)$ & $2 / 79(3 \%)^{*}$ & $>0.99$ \\
\hline \multicolumn{4}{|l|}{ ARDS treatment during ICU stay } \\
\hline Corticosteroids (any dose) & $24(63 \%)$ & $32(41 \%)^{*}$ & 0.02 \\
\hline Corticosteroids (low dose) & $22(58 \%)$ & $22(28 \%)^{*}$ & 0.002 \\
\hline Corticosteroids (high dose) \# & $2(5 \%)$ & $10(13 \%)^{*}$ & 0.22 \\
\hline Prone position & $20(51 \%)$ & $71(88 \%)$ & $<0.0001$ \\
\hline
\end{tabular}


Table 1 (continued)

\begin{tabular}{|c|c|c|c|}
\hline & NC-ARDS $(n=39)$ & C-ARDS $(n=81)$ & p-value \\
\hline Neuromuscular blockade & $25(64 \%)$ & $74(91 \%)$ & $<0.0001$ \\
\hline Inhaled nitric oxide & $6(15 \%)$ & $28(35 \%)$ & 0.03 \\
\hline Extra-corporeal membrane oxygenation & $5(13 \%)$ & $20(25 \%)$ & 0.13 \\
\hline \multicolumn{4}{|l|}{ Organ support and outcome during ICU stay } \\
\hline Renal replacement therapy during ICU stay & $19(49 \%)$ & $29(36 \%)$ & 0.18 \\
\hline Norepinephrine, n (\%) & $32(82 \%)$ & $61(75 \%)$ & 0.41 \\
\hline ICU length of stay among survivors, days & 17 [10-28] & $30[22-46]$ & 0.09 \\
\hline Death at day 28 & $15(39 \%)$ & $30(37 \%)$ & 0.88 \\
\hline Death in the ICU & $17(44 \%)$ & $32(40 \%)$ & 0.67 \\
\hline \multicolumn{4}{|l|}{ Pneumocystis jirovecii samples and analysis } \\
\hline Total samples, mean (range) & $1.5(1-4)$ & $3.8(1-15)$ & $<0.001$ \\
\hline Sputum examination, mean (range) & $0.08(0-1)$ & 0 & 0.01 \\
\hline Broncho-alveolar lavage, mean (range) & $1.5(0-4)$ & $0.19(0-2)$ & $<0.001$ \\
\hline Blind protected sample, mean (range) & 0 & $3.6(1-15)$ & $<0.001$ \\
\hline Direct examination (IF or MGG) & $1.5(0-4)$ & $0.19(0-2)$ & $<0.001$ \\
\hline $\mathrm{qPCR}$ & $1.5(1-4)$ & $3.8(1-15)$ & $<0.001$ \\
\hline Serum (1-3)-BDG & $0.5(0-4)$ & $4(1-10)$ & $<0.001$ \\
\hline
\end{tabular}

COPD = chronic obstructive pulmonary disease, HIV = human immunodeficiency virus, SAPS II= Simplified Acute Physiology Score II, SOFA = sequential organ failure assessment, ICU = intensive care unit; *two missing values because two patients received dexamethasone or placebo in a randomized controlled trial; \#denotes more than $1 \mathrm{mg} / \mathrm{kg}$ of prednisone or equivalent

Table 2 Characteristics of patients with a positive Pneumocystis jirovecii qPCR

\begin{tabular}{|c|c|c|c|c|c|c|c|c|c|}
\hline $\begin{array}{l}\text { Patient, } \\
\text { age, sex }\end{array}$ & $\begin{array}{l}\text { Underlying } \\
\text { disease }\end{array}$ & $\begin{array}{l}\text { Date of PCP } \\
\text { diagnosis }\end{array}$ & $\begin{array}{l}\text { Viral } \\
\text { association }\end{array}$ & $\begin{array}{l}\text { Respiratory } \\
\text { sample }\end{array}$ & $\begin{array}{l}\text { Direct } \\
\text { examination } \\
\text { (IFI or MGG) }\end{array}$ & $\begin{array}{l}\text { Pneumocystis } \\
\text { qPCR }\end{array}$ & $\begin{array}{l}\text { BDG (pg/ } \\
\mathrm{ml})^{*}\end{array}$ & $\begin{array}{l}\text { Time } \\
\text { between } \\
\text { ICU } \\
\text { admission } \\
\text { and } \\
\text { positive } \\
\text { sample } \\
\text { (day) }\end{array}$ & Treatment \\
\hline P1, 58y, M & $\begin{array}{l}\text { Diabetus } \\
\text { mellitus } \\
\text { Congestive } \\
\text { heart failure }\end{array}$ & $14 / 01 / 2014$ & $\begin{array}{l}\text { Coronavirus, } \\
\text { Rhinovirus }\end{array}$ & $\mathrm{BAL}$ & Negative & 36.7 & NA & 1 & No \\
\hline$P 2,73 y, M$ & $\begin{array}{l}\text { Renal trans- } \\
\text { plantation } \\
\text { Diabetus } \\
\text { mellitus } \\
\text { Congestive } \\
\text { heart failure }\end{array}$ & $29 / 08 / 2015$ & Coronavirus & $B A L$ & Positive & 32 & 106 & 0 & $\begin{array}{l}\text { Yes } \\
\text { (sulfameth- } \\
\text { oxazole) }\end{array}$ \\
\hline P3, 52y, M & $\begin{array}{l}\text { Myasthenia } \\
\text { (steroid, aza- } \\
\text { thioprine) }\end{array}$ & 04/07/2012 & $\begin{array}{l}\text { Respiratory } \\
\text { syncytial } \\
\text { virus }\end{array}$ & $B A L$ & Negative & 39.8 & NA & 1 & No \\
\hline$P 4,32 y, F$ & $\begin{array}{l}\text { Acute } \\
\text { lymphoblas- } \\
\text { tic leukemia } \\
\text { (methotrex- } \\
\text { ate and } \\
\text { aracytabine) }\end{array}$ & 08/01/2019 & $\begin{array}{l}\text { Metapneu- } \\
\text { movirus }\end{array}$ & BAL & Positive & 27.9 & 188 & 0 & $\begin{array}{l}\text { Yes } \\
\text { (sulfameth- } \\
\text { oxazole) }\end{array}$ \\
\hline$P 5,67 y, M$ & $\begin{array}{l}\text { Cirrhosis, } \\
\text { rheumatoid } \\
\text { polyarthritis } \\
\text { (steroid) }\end{array}$ & 22/04/2019 & $\begin{array}{l}\text { Coronavirus } \\
\text { NL63 }\end{array}$ & BAL & Negative & 36.6 & NA & 1 & No \\
\hline
\end{tabular}

$\mathrm{M}=$ male, $\mathrm{F}=$ female, $\mathrm{P}=$ patient; $\mathrm{BDG}=(1-3)-\beta-\mathrm{D}$-glucan, ${ }^{*}$ BDglucan not performed in the lab before 2013. BDglucan was performed using the Fungitell kit ${ }^{\mathrm{TM}}$ (Cape Cod Inc, USA) with a positivity threshold of $80 \mathrm{pg} / \mathrm{mL} ; \mathrm{qPCR}$ of P.jirovecii was performed using a region of the mitochondrial large subunit rRNA gene (LSU) after DNA extraction with a Qiasymphony kit (Qiagen, Courtaboeuf, France) 
one positive PCR, with a median cycle threshold of 36.6 [30-38.3].

Two NC-ARDS patients fulfilled proven PCP diagnostic criteria, with a positive direct examination, a single $ß$-D-glucan $>80 \mathrm{pg} / \mathrm{mL}$ (Table 2), and received treatment for PCP.

Three other NC-ARDS patients were classified as colonized, while no patient fulfilled possible PCP diagnostic criteria. Time between ICU admission and positive sample for PCP (Table 2) was short ( $<2$ days) like in other invasive fungal infections (i.e. invasive pulmonary aspergillosis) in severe influenza infection or ARDS.

In this study of patients with viral ARDS, we found a low risk for possible or proven PCP. Our findings are in accordance with two smaller studies in France $[4,5]$ retrieving a low risk of Pneumocystis colonisation in COVID-19 patients. In our cohort, qPCR was positive in $13 \%$ of NC-ARDS. This result is in accordance with a previous study showing $7 \%$ of positive qPCR in ICU-admitted influenza patients [6].

The strengths of our study are the analysis of a large ARDS cohort with fungal analyses. Our study also has limitations: monocentric design, NC-ARDS patients more frequently immunocompromised, and a long cohort period.

\section{Abbreviations \\ C-ARDS: Coronavirus disease 19 related acute respiratory distress syndrome; NC-ARDS: Non-coronavirus disease 19 viral ARDS; PCP: Pneumocystis jirovecii Pneumonia.}

\section{Acknowledgements}

We thank Jean Hazebroucq and Francois Hemery, for their invaluable help. We are very indebted for the COVID-PCP group for the help in the data management and the review of the final manuscript. The COVID PCP group is composed by the followings members: Slim Fourati, Brice Benelli, Frédérique Boquel, Nicolas de Prost, Guillaume Carteaux, Jeanne Tran Van Nhieu, Frederic schlemmer.

\section{Authors' contributions \\ $\mathrm{KR}, \mathrm{RA}$ and FB contributed to the study design, analysis and interpretation of data. KR, RA and AMD drafted the initial manuscript and approved the article final version. AFH, SF, AMD and the COVID-PCP group contributed to the interpretation of data, critical revision of intellectual content and approval of the submitted version of the article. All authors read and approved the final manuscript.}

\section{Funding}

No funding was received for this study.

\section{Declarations}

Ethics approval and consent to participate

This observational study was approved by the Ethical Review Board of the French Society for Intensive Care Medicine (Société de Réanimation de Langue Française). As per the French law, no informed consent was required for this type of studies.

\section{Consent for publication}

Not applicable.

\section{Competing interests}

All authors report no conflict of interest relevant to this study.

\section{Author details}

${ }^{1}$ Service de Médecine Intensive Réanimation, AP-HP (Assistance PubliqueHôpitaux de Paris), Hôpitaux Universitaires Henri Mondor, DMU Médecine, 94010 Créteil, France. ${ }^{2}$ Faculté de Santé de Créteil, IMRB, GRC CARMAS, UPEC (Université Paris Est Créteil), 94010 Créteil, France. ${ }^{3}$ Département de Virologie, Bactériologie, Parasitologie-Mycologie, AP-HP (Assistance Publique-Hôpitaux de Paris), Hôpitaux Universitaires Henri Mondor, 94010 Créteil, France. ${ }^{4}$ EA 7380 Dynamic, UPEC, Ecole Nationale Vétérinaire d'Alfort, USC Anses, Créteil, France. ${ }^{5}$ INSERM, Unité U955, France Université Paris Est, 94010 Créteil, France. ${ }^{6}$ Service de Medecine Intensive Réanimation, CHU Henri Mondor, 51, Av de Lattre de Tassigny, 94000 Créteil Cedex, France.

Received: 16 August 2021 Accepted: 11 September 2021

Published online: 26 September 2021

\section{References}

1. Razazi K, Arrestier R, Haudebourg AF, Benelli B, Carteaux G, Decousser J-W, et al. Risks of ventilator-associated pneumonia and invasive pulmonary aspergillosis in patients with viral acute respiratory distress syndrome related or not to Coronavirus 19 disease. Crit Care Lond Engl. 2020;24:699.

2. Botterel F, Cabaret O, Foulet F, Cordonnier C, Costa J-M, Bretagne S. Clinical significance of quantifying Pneumocystis jirovecii DNA by using realtime PCR in bronchoalveolar lavage fluid from immunocompromised patients. J Clin Microbiol. 2012;50:227-31.

3. Donnelly JP, Chen SC, Kauffman CA, Steinbach WJ, Baddley JW, Verweij PE, et al. Revision and update of the consensus definitions of invasive fungal disease from the european organization for research and treatment of cancer and the mycoses study group education and research consortium. Clin Infect Dis Off Publ Infect Dis Soc Am. 2020;71:1367-76.

4. Blaize M, Mayaux J, Luyt C-E, Lampros A, Fekkar A. COVID-19-related respiratory failure and lymphopenia do not seem associated with pneumocystosis. Am J Respir Crit Care Med. 2020;202:1734-6.

5. Alanio A, Dellière S, Voicu S, Bretagne S, Mégarbane B. The presence of Pneumocystis jirovecii in critically ill patients with COVID-19. J Infect. 2020;

6. Beumer MC, Koch RM, van Beuningen D, OudeLashof AM, van de Veerdonk FL, Kolwijck E, et al. Influenza virus and factors that are associated with ICU admission, pulmonary co-infections and ICU mortality. J Crit Care. 2019;50:59-65.

\section{Publisher's Note}

Springer Nature remains neutral with regard to jurisdictional claims in published maps and institutional affiliations. 